Disclosure of Interest: None declared

DOI: 10.1136/annrheumdis-2018-eular.4600

\section{THU0420 $\quad$ AN EXTENT OF INTERSTITIAL LUNG DISEASE IS A POTENTIAL PREDICTOR OF RESPONSE TO A-B-CELL THERAPY IN THE PATIENTS WITH SSC}

O. Koneva, O. Desinova, O. Ovasyannikova, L. Garzanova, M. Starovoytova, L. Ananieva. Nasonova Research Institute of Rheumatology, Moscow, Russian Federation

Background: Systemic sclerosis (SSc) is a connective tissue disease associated with chronic polyclonal B-lymphocytic activation and immunological tolerance disturbance. Several research and clinical studies showed that B-cell depletion is potentially efficacious in SSc treatment. However, neither strong evidence of RTX efficacy for treatment of interstitial lung disease (ILD) associated with SSc, no potential predictor of response to a-B-cell therapy.

Objectives: To evaluate rituximab (RTX) therapy efficacy in the patients with systemic scleroderma (SSc) differing in extent of interstitial lung disease (ILD) based on miltispiral computed tomography (MSCT) findings.

Methods: 42 patients (average age $48 \pm 2$ years; male/female 1/6, diffuse/limited disease 1.5/1 (25 and 17), disease duration since the first non-Raynaud syndrome $-6.6 \pm 5.9$ years) with definitely diagnosed SSc and ILD signs evidenced by MSCT were enrolled into the study. During the observation period $29 \pm 15.3$ months the patients received rituximab (RTX) total dose of $2.5 \pm 1.3$ grams in combination with glucocorticoids at average dose of $11.7 \pm 3.9 \mathrm{mg}$. 10 (24\%) patients concurrently took immunosuppresants. The therapy efficacy was evaluated both in the general study population and in the patient subgroups with interstitial lesion extent up to $20 \%$ (Group $A, n=13$ ) and greater than $20 \%$ (Group B, $n=29$ ) of total pulmonary tissue area. ${ }^{1}$

Results: In the general patient population significant FVC increase from 73.2 $\pm 18.8 \%$ to $82 \pm 21.8 \%(\mathrm{p}=0.000031)$ and stabilisation of DLCO $(42.6 \% \pm 15.7 \%$ vs $44.7 \pm 14.6 \%$. $p=0.2)$ were observed. Median FVC increment was $6 \%(25$ th $\%$ $>3,3 \% ; 75$ th $\%>16 \%)$. FVC-based parameters increased by $\geq 10 \%$ in $16(38 \%)$ patients and decreased in $3(7 \%)$ patients.

Average FVC values in Group A were significantly higher compared with Group B both at the baseline $(88.8 \% \pm 18.6 \%$ vs $65.4 \pm 14.5 \%, p=0.0002)$ and after the treatment $(103.3 \% \pm 15.9 \%$ vs $74.1 \pm 18.5 \%, p=0.0009)$ with statistically significant FVC increase in both groups during the treatment period $(p=0.016$ and $p=0.0014$, respectively). Median FVC increment in Group A and Group B was $10.2 \%$ (25th\% $>4.7 \%$; 75th $\%>21.9 \%$ ) and $5.9 \%$ (25th\%>2.75\%; 75th\%>14.7\%), p>0.05, respectively. FVC-based parameters increased by $\geq 10 \%$ in 6 (46\%) patients in Group A and in $10(34 \%)$ patients in Group B, and decreased in $1(8 \%)$ and $2(7 \%)$ patients, respectively

Average DLCO values were also significantly higher in Group A compared to Group B both before and after treatment $(58.4 \% \pm 16.4 \%$ vs $36.3 \pm 10.1 \%$. $p=0.025$; $59.3 \pm 15.2 \%$ vs $38.9 \pm 9.7 \%$. $p=0.005$ ); DLCO values did not change over time during RTX therapy.

Conclusions: RTX therapy resulted in significant FVC increase. FVC increment in the patient group with ILD extent up to $20 \%$ achieved clinical significance level in contrast to the patients with ILD extent greater than $20 \%$, where FVC increment was $5.9 \%$. Obtained data suggest that initial lung lesion area is a potential predictor of response to a-B-cell therapy in the patients with SSc.

\section{REFERENCE:}

[1] Moore O, Goh N, Corte T, et al. Extent of disease on high- resolution computed tomography lung is a predictor of decline and mortality in systemic sclerosis -related interstitial lung disease. Rheumatology. 2013; 52:155160. doi:10.1093/rheumatology/kes289

Disclosure of Interest: None declared

DOI: 10.1136/annrheumdis-2018-eular.4543

\section{THU0421 PREVALENCE AND CLINICAL CORRELATES OF SMALL AIRWAY OBSTRUCTION IN PATIENTS WITH SYSTEMIC SCLEROSIS}

P. Ostojic $^{1,2}$, M. Vujovic ${ }^{2}$, A. Blanka Protic ${ }^{3} .{ }^{1}$ Institute of Rheumatology; ${ }^{2}$ School of Medicine, University of Belgrade; ${ }^{3}$ Clinical Center of Serbia, Clinic for Pulmology, Belgrade, Serbia

Background: Small airways are usually defined as non-cartilaginous airways with an internal diameter $<2 \mathrm{~mm}$. Small airway obstruction (SAO) may be result of a primary bronchiolar disorder, or secondary to a disease that also affects large airways (like asthma or chronic obstructive pulmonary disease - COPD), or related to an interstitial lung disease with bronchiolar involvement.
Objectives: To assess prevalence and clinical correlates of SAO in patients with systemic sclerosis (SSc).

Methods: 69 consecutive patients with SSc (63 women and 6 men) were included in this study. Patients with previously diagnosed bronchiectasis, COPD or asthma were excluded. Forty two (60.9\%) patients had limited cutaneous SSc whilst $27(39.1 \%)$ of patients had diffuse form of the disease. Seventeen patients $(24.6 \%)$ were tobacco-smokers, $52(75.4 \%)$ were nonsmokers. Lung function tests, including assessment of lung diffusing capacity, were performed in al patients. Patients were considered to have small airway obstruction (SAO) when Maximum Expiratory Flow at $25 \%$ of the forced vital capacity $\left(\mathrm{MEF}_{25}\right)$ was lower than $60 \%$ as predicted. We assessed the relationship of SAO in our patients with large airway obstruction, decreased lung diffusing capacity, disease duration, dis ease subtype, scleroderma-specific antibodies and smoking.

Results: SAO was noticed in 46/69 (66.6\%) of patients with SSc. Restrictive lung disease was found in 4/69 (5.8\%), obstruction of large airways in 18/69 (26.1\%) and decreased lung diffusing capacity in 47/69 (68.1\%) of patients. No difference in gender, age, disease duration, disease form and scleroderma-specific antibodies was found between patients with and without SAO. 18/46 (39.1\%) patients with SAO had decreased $F E V_{1}$ and $F E V_{1} / F V C$, indicating presence of coexistent large airway obstruction. Indeed, all patients with signs of obstructive lung disease on spirometry, had associated SAO. Moreover, $\mathrm{MEF}_{25}$ correlated significantly with FEV FE $_{1}(\rho=0.54, \mathrm{p}<0.001), \mathrm{FEV}_{1} / \mathrm{FVC}(\rho=0.74, \mathrm{p}<0.001), \operatorname{PEF}(\rho=0.29, \mathrm{p}=0.02$ and $\operatorname{MEF}_{50}(\rho=0.80, p<0.001)$ in our patients with SSc. However, $28 / 46(60.9 \%)$ SSc patients with SAO did not have signs of concomitant large airway obstruction. 20/46 (43.5\%) patients had associated decreased lung diffusing capacity, whils $8 / 46(17.4 \%)$ of patients had isolated SAO. We have found that tobacco-smokers among SSc patients with SAO have more common associated obstructive lung disease on spirometry ( $58.8 \%$ vs $15.4 \%, p=0.004)$, and have a significantly lower mean value of $\mathrm{MEF}_{25}$ compared to non-smokers $(32.1 \% \pm 7.1 \%$ vs. $45.4 \pm 11.6 \%$, $\mathrm{p}=0.001$ ). On the other hand, isolated SAO, and SAO associated with impaired diffusing capacity was equally frequent among smokers and non-smokers.

Conclusions: Patients with SSc have commonly SAO. It can be considered as clinical feature of undiagnosed asthma or COPD, if associated with large airway obstruction, especially in tobacco-smokers. On the other hand, isolated SAO or associated with decreased lung diffusing capacity was found to be not related to smoking, and may indicate a possible prominent bronchiolar involvement within SSc related interstitial lung disease.

Disclosure of Interest: None declared

DOI: 10.1136/annrheumdis-2018-eular.3944

\section{THU0422 PERFORMANCE OF EULAR/ACR 2017 IDIOPATHIC INFLAMMATORY MYOPATHIES CLASIFICATION CRITERIA IN A REAL WORLD COHORT}

R.A. Gómez, A. Braillard Poccard, M. Pino, G. Medina, D. Dubisnky. HTAL DE CLÍNICAS, Ciudad Autonoma de Buenos Aires, Argentina

Background: Idiopathic Inflammatory Myopathies (IIM) are an heterogeneous group of multisystemic diseases. It includes Polimyositis (PM), Dermatomyositis (DM) with its clinicaly amiopathic variant (CADM), the antisynthetase syndrome (ASS), the inclusion body myositis (IBM), Immune-mediated necrotising myopathy (IMNM), the juvenile variants of DM/PM and the connective tissue disease myosits overlap (CTD-OM).

Distintion between subtypes is made on grounds of clinical features, histologic findings at muscle biopsy and presence of certain autoantibodies.

Multiple classification criteria had been proposed through time, EULAR/ACR been the most recent. However, their performance in patients from common prac tice in Latin America had not been widely evaluated.

In our practice, access to muscle biopsy and electromyogram (EMG) is not always available

Objectives: To evaluate the performance of the EULAR/ACR 2017 IIM classification criteria in a real world cohort and compare it with the performance of other classification criteria

Methods: Retrospective study. IIM patients defined by expert opinion followed in our centre between October 2007 and November 2017 were included.

The patients were classified clinically in DM, CADM, PM, ASS and CTD-OM Patients with positive antisynthetase antibodies were reclassified as ASS.

Availability of EMG, muscle biopsy and anti Jo-1 antibodies was evaluated. Bohan \& Peter (1975) Tanimoto (1995) y EULAR/ACR (2017) criteria were applied to the population

Results: 60 patients were included. DM 20 (33.3\%), CADM 4 (6.66\%), PM 4 $(6.66 \%)$, ASS $10(16.66 \%)$ y CTD-OM 22 (36.66\%). Muscle biopsy available 14 $60(23.33 \%)$, EMG available $33 / 60(55 \%)$ and anti Jo-1 determination available in $57 / 60(95 \%)$.

In general, 12/60 (20\%) classified as defined disease by Bohan and Peter criteria $29 / 60(48.3 \%)$ by Tanimoto criteria and 34/60 (56.6\%) by EULAR/ACR 2017 criteria. 